\title{
Exploring the association of Fermi sources with Young Stellar Objects
}

\author{
P. Munar-Adrover ${ }^{1}$, J. M. Paredes ${ }^{1}$ and G. E. Romero ${ }^{2}$ \\ ${ }^{1}$ Dept. d'Astronomia i Meteorologia, Universitat de Barcelona. email: pmunar@am.ub.es \\ ${ }^{2}$ Instituto Argentino de Radioastronomía, Universidad de La Plata
}

\begin{abstract}
Massive protostars have associated bipolar outflows which can produce strong shocks when interact with the surrounding medium. Some theoretical models predict that particle acceleration at relativistic velocities can occur leading to gamma ray emission. In order to identify young stellar objects (YSO) that might emit gamma rays, we have crossed the Fermi First Year Catalog with catalogs of known YSOs, obtaining a set of candidates by spatial correlation. We have conducted Montecarlo simulations to find the probability of chance coincidence. Our results indicate that $\sim 70 \%$ of the candidates should be gamma-ray sources with a confidence of $\sim 5 \sigma$.
\end{abstract}

Keywords. stars: early-type, gamma rays: observations, ISM: jets and outflows

\section{Introduction}

Massive YSOs show collimated outflows and thermal radiation has been detected up to distances of $10^{16}-10^{18} \mathrm{~cm}$ from the central star. These are strongly supersonic jets and in some cases, non-thermal radio lobes have been detected at distances of $Z_{j} \sim 1 \mathrm{pc}$ (Garay et al. 2003). These radio lobes are probably generated by strong terminal shocks of the jets, which also ionize the shocked material. The possibility of YSOs to be $\gamma$-ray emitters has already been discussed in Araudo et al. (2007) and Bosch-Ramon et al. (2010). The action of the jet head on the external medium leads two shocks, the bow shock and the reverse shock. The observed non-thermal radio emission would be generated at the shocks where the particles are accelerated.

\section{Numerical simulations}

With the aim of finding the positional coincidences between Fermi sources and Young Stellar Objects we have crossed the First Fermi-LAT Catalog (1FGL) (Abdo et al. 2010) and a catalog of MYSOs from the RMS Survey (Urquhart et al. 2009) by using a computer code that determines the angular distance between two points in the sky, taking into account the positional uncertainties in each of them. We ran the code with the 1392 sources of the 1FGL that have not been firmly identified, and the 556 sources identified as YSOs in the RMS survey. In order to estimate the statistical significance of these coincidences, we have simulated a large number of sets of Fermi sources. Specifically, we have simulated 1500 populations of 1392 Fermi sources, through rotations on the celestial sphere, displacing a source with original galactic coordinates $(l, b)$ to a new position $\left(l_{0}, b_{0}\right)$ as done in Romero et al. (1999). The separation between the Fermi source and the YSO is calculated in each case using the statistical parameter $R$ (Allington-Smith et al. 1982)

$$
R=\sqrt{\frac{(\Delta \alpha \cos \delta)^{2}}{\sigma_{i_{\alpha}}^{2}+\sigma_{j_{\alpha}}^{2}}+\frac{\Delta \delta^{2}}{\sigma_{i_{\delta}}^{2}+\sigma_{j_{\delta}}^{2}}}
$$

where $\sigma_{\alpha_{i}}, \sigma_{\delta_{i}}$ is the uncertainty in the position of the source, and $i$ and $j$ are Fermi and RMS sources, respectively. 


\section{Results}

We have found 13 Fermi sources being positionally coincident with 24 YSOs (see Table 1), and 8 of these Fermi sources have not any proposed counterpart (like SNR, PWN, pulsar, etc.) to the gamma emission. In Table 2 we present the statistical results obtained from simulations with a random distribution in galactic longitude. As can be seen, the estimated probability of a pure chance association is as low as $2.0 \times 10^{-8}$ for the $2^{\circ}$-binning simulations $\left(2.2 \times 10^{-6}\right.$ for the $1^{\circ}$-binning $)$, and there is a correlation at $\sim 5 \sigma$. When we considered the restrictions in both $l$ and $b$ (see Table 3), the chance probability raised, but still shows a quite negligible values $\left(\sim 10^{-6}\right)$.

\begin{tabular}{|c|c|c|c|c|c|c|c|c|}
\hline Fermi Name & $\begin{array}{l}95 \% \text { Semi } \\
\text { Major Axis }\end{array}$ & $\begin{array}{l}\text { Spectral Index } \Gamma \\
\qquad F \propto E^{-\Gamma}\end{array}$ & $\begin{array}{l}\text { Energy Flux } \\
\mathrm{erg} \mathrm{cm}^{-2} \mathrm{~s}^{-1}\end{array}$ & MSX Name & $\begin{array}{l}\text { Freq. } \\
\mathrm{GHz}\end{array}$ & $\begin{array}{l}\text { Int.Flux } \\
\text { mJy }\end{array}$ & $\underset{\circ}{\text { Angular dist. }}$ & $\mathrm{R}$ \\
\hline 1FGL J0541.1+3542 & 0.1397 & $2.41 \pm 0.13$ & $1.61 \times 10^{-11} \pm 4.9 \times 10^{-12}$ & $\begin{array}{l}\text { G173.6328+02.8064 } \\
\text { G173.6339+02.8218 } \\
\text { G173.6882+02.7222 } \\
\text { G173.7215+02.6924 }\end{array}$ & $\begin{array}{l}5 \\
5 \\
5 \\
5\end{array}$ & $\begin{array}{l}<0.7 \\
<0.7 \\
<0.8 \\
<0.8\end{array}$ & $\begin{array}{l}0.12 \\
0.13 \\
0.05 \\
0.05\end{array}$ & $\begin{array}{l}0.846 \\
0.903 \\
0.364 \\
0.386\end{array}$ \\
\hline 1FGL J0647.3+0031 & 0.2150 & $2.41 \pm 0.11$ & $1.89 \times 10^{-11} \pm 5.4 \times 10^{-12}$ & G212.0641-00.7395 & 5 & $<0.9$ & 0.10 & 0.467 \\
\hline 1FGL J1256.9-6337 & 0.1955 & $2.26 \pm 0.12$ & $4.97 \times 10^{-11} \pm 1.1 \times 10^{-11}$ & G303.5990-00.6524 & 4.8 & $<0.6$ & 0.12 & 0.589 \\
\hline 1FGL J1315.0-6235 & 0.1860 & $2.31 \pm 0.12$ & $6.86 \times 10^{-11} \pm 0.0$ & $\begin{array}{l}\text { G305.4840+00.2248 } \\
\text { G305.5610+00.0124 }\end{array}$ & $\begin{array}{l}8.6 \\
8.6\end{array}$ & $\begin{array}{l}<2.1 \\
7.2\end{array}$ & $\begin{array}{l}0.18 \\
0.16\end{array}$ & $\begin{array}{l}0.958 \\
0.869\end{array}$ \\
\hline 1FGL J1651.5-4602 & 0.2258 & $2.21 \pm 0.07$ & $1.39 \times 10^{-10} \pm 3.4 \times 10^{-11}$ & G339.8838-01.2588 & 8.6 & 2.6 & 0.14 & 0.638 \\
\hline 1FGL J1702.4-4147 & 0.0800 & $2.39 \pm 0.07$ & $8.7 \times 10^{-11} \pm 2.0 \times 10^{-11}$ & $\begin{array}{l}\text { G344.4257+00.0451B } \\
\text { G344.4257+00.0451C }\end{array}$ & $\begin{array}{l}8.6 \\
8.6\end{array}$ & $\begin{array}{l}<13.7 \\
<13.7\end{array}$ & $\begin{array}{l}0.05 \\
0.05\end{array}$ & $\begin{array}{l}0.631 \\
0.638\end{array}$ \\
\hline 1FGL J1846.8-0233 & 0.1262 & $2.21 \pm 0.06$ & $9.3 \times 10^{-11} \pm 2.3 \times 10^{-11}$ & G030.1981-00.1691 & 5 & $<0.8$ & 0.08 & 0.646 \\
\hline 1FGL J1848.1-0145 & 0.0859 & $2.23 \pm 0.04$ & $9.5 \times 10^{-11} \pm 3.2 \times 10^{-11}$ & $\begin{array}{l}\text { G030.9726-00.1410 } \\
\text { G030.9959-00.0771 }\end{array}$ & $\begin{array}{l}5 \\
5 \\
\end{array}$ & $\begin{array}{l}<0.7 \\
<1.2\end{array}$ & $\begin{array}{l}0.07 \\
0.00\end{array}$ & $\begin{array}{l}0.763 \\
0.036\end{array}$ \\
\hline 1FGL J1853.1+0032 & 0.5207 & $2.18 \pm 0.07$ & $5.7 \times 10^{-11} \pm 1.7 \times 10^{-11}$ & $\begin{array}{l}\text { G032.8205-00.3300 } \\
\text { G033.3891+00.1989 } \\
\text { G033.3933+00.0100 } \\
\text { G034.0126-00.2832 } \\
\text { G034.0500-00.2977 }\end{array}$ & $\begin{array}{l}5 \\
5 \\
5 \\
5 \\
5\end{array}$ & $\begin{array}{l}<0.7 \\
<1.0 \\
<1.1 \\
<0.7 \\
<0.8\end{array}$ & $\begin{array}{l}0.34 \\
0.40 \\
0.26 \\
0.43 \\
0.47\end{array}$ & $\begin{array}{l}0.658 \\
0.768 \\
0.496 \\
0.830 \\
0.908\end{array}$ \\
\hline 1FGL J1925.0+1720 & 0.1443 & $2.28 \pm 0.12$ & $2.37 \times 10^{-11} \pm 1.02 \times 10^{-11}$ & $\begin{array}{c}\mathrm{G} 052.2078+00.6890 \\
\mathrm{G} 052.2025+00.7217 \mathrm{~A}\end{array}$ & $\begin{array}{l}5 \\
5\end{array}$ & $\begin{array}{l}<0.7 \\
<0.8\end{array}$ & $\begin{array}{l}0.08 \\
0.07\end{array}$ & $\begin{array}{l}0.533 \\
0.453\end{array}$ \\
\hline 1FGL J1943.4+2340 & 0.1118 & $2.23 \pm 0.11$ & $2.62 \times 10^{-11} \pm 6.8 \times 10^{-12}$ & G059.7831+00.0648 & 8.6 & 1.0 & 0.08 & 0.743 \\
\hline 1FGL J2032.8+3928 & 0.2507 & $2.59 \pm 0.07$ & $5.1 \times 10^{-11} \pm 1.4 \times 10^{-11}$ & G078.4705-00.1830 & 5 & $<1.2$ & 0.23 & 0.908 \\
\hline 1FGL J2040.0+4157 & 0.1970 & $2.66 \pm 0.06$ & $7.9 \times 10^{-11} \pm 1.2 \times 10^{-11}$ & G081.5168+00.1926 & 5 & $<1.1$ & 0.04 & 0.195 \\
\hline
\end{tabular}

Table 1. Positional coincidence between Fermi sources and MYSOs.

\begin{tabular}{ccccc}
\hline $\begin{array}{c}\text { Actual } \\
\text { coincidence }\end{array}$ & $\begin{array}{c}\text { Simulated } \\
1^{\circ} \text {-bin }\end{array}$ & $\begin{array}{c}\text { Probability } \\
1^{\circ}\end{array}$ & $\begin{array}{c}\text { Simulated } \\
2^{\circ} \text {-bin }\end{array}$ & $\begin{array}{c}\text { Probability } \\
2^{\circ}\end{array}$ \\
\hline 13 & $3.9 \pm 1.9$ & $2.2 \times 10^{-6}$ & $3.3 \pm 1.7$ & $2.0 \times 10^{-8}$ \\
\hline
\end{tabular}

Table 2. Statistical results obtained from simulations with a random distribution in galactic longitude.

\begin{tabular}{ccccc}
\hline \hline $\begin{array}{c}\text { Actual } \\
\text { coincidence }\end{array}$ & $\begin{array}{c}\text { Simulated } \\
20^{\circ} \text {-bin }\end{array}$ & $\begin{array}{c}\text { Probability } \\
20^{\circ}\end{array}$ & $\begin{array}{c}\text { Simulated } \\
40^{\circ} \text {-bin }\end{array}$ & $\begin{array}{c}\text { Probability } \\
40^{\circ}\end{array}$ \\
\hline 13 & $5.17 \pm 2.07$ & $1.5 \times 10^{-4}$ & $3.8 \pm 1.9$ & $1.6 \times 10^{-6}$ \\
\hline
\end{tabular}

Table 3. Statistical results obtained from simulations constrained both in galactic latitude and galactic longitude.

Acknowledgements. J.M.P. and G.E.R. acknowledge support by DGI of the Spanish Ministerio de Educación y Ciencia (MEC) under grant AYA2007-68034-C03-01 and FEDER funds. In addition, this work has been supported by the Consejería de Innovación, Ciencia y Empresa (CICE) of Junta de Andalucía as research group FQM-322 and excellence grant FQM-5418.

\section{References}

Abdo A. A. et al., 2010, ApJ Supplement Series, 188, 405-436

Allington-Smith J. R., Perryman M. A. C., Longair M. S. et al., 1982, MNRAS, 201, 331-344

Araudo A. T., Romero G. E., Bosch-Ramon V., and Paredes J. M., 2007, A\&A, 476, 1289-1295

Bosch-Ramon V., Romero G. E., Araudo A. T. and Paredes J. M., 2010, AESA, 511, A8

Garay G., Brooks K. J., Mardones D., and Norris R. P., 2003, ApJ, 587, 739-747

Romero G. E., Benaglia P. and Torres D. F., 1999, A\&AA, 348, 868-876

Urquhart J. S., Horae M. G., Purcell C. R. et al., 2009, A\& A, 501, 539-551 